\title{
Signatures of black holes in string theory
}

\author{
Metin Gürses* \\ Department of Mathematics, Faculty of Science, Bilkent University, 06533 Ankara, Turkey \\ (Received 20 April 1992)
}

\begin{abstract}
The effect of string theory on the four-dimensional classical Einstein equations is investigated. It is shown that the throats of nonrotating charged black holes are exact solutions of the gravitational field equations with string correction terms.
\end{abstract}

PACS number(s): 11.17. $+\mathrm{y}, 04.20 . J b, 04.60 .+\mathrm{n}$

Gravitational field equations with string correction terms have recently drawn much attention in several respects. The most important contribution of these terms is believed to change the singularity structure of the spacetime geometry. For this purpose there has been much interest in the black-hole solutions [1-10] of the higher-dimensional Einstein field equations containing higher-order curvature terms. Although not yet known, it is believed that the low-energy string action is a perturbation expansion in inverse powers of the string tension parameter. This expansion contains, in addition to the usual Einstein-Hilbert action, corrections quadratic and higher-order invariants in the massless fields, curvature tensor, and in Maxwell and dilaton fields.

Recently it has been shown that the plane-wave metrics of the Einstein theory preserve their form under string corrections at all orders [11-13]. The question arises as to whether or not there exist spherically symmetric spacetimes with the same property. In particular, nonrotating black-hole geometries are spherically symmetric and it is believed that they do not preserve their form [1].

Since gravitation is weak far from the holes, the blackhole solutions of the Einstein theory can be considered as approximate solutions of the effective field theory mentioned above. On the other hand, near the singularities, the contribution of curvature terms in the extended theory becomes important. Black-hole solutions of the Einstein theory are not any more exact solutions of the extended theory in these regions. In the general case, it is very hard, almost impossible, to consider the full extended field equations and find their exact solutions with the property that they asymptotically approach the blackhole solutions of the Einstein theory. Recently [9] it has been conjectured that metrics describing the neighborhood of the event horizon of the extreme charged black holes may solve the extended field equations exactly. Such candidates are of extreme Reissner-Nordström type and the recently found metric with a dilaton charge $[8,9]$. In this paper we shall show that the throats of these black-hole solutions are, in fact, exact solutions of the extended field equations.

\footnotetext{
${ }^{*}$ Electronic address: Gurses at trbilun.
}

We assume a flat internal space, an Abelian gauge field with zero components in the internal directions, and set the three-form field equal to zero. We also assume that the four-dimensional metric, the Maxwell, and dilaton fields do not depend on the internal coordinates. With these assumptions the four-dimensional low-energy action obtained from string theory is [9]

$$
\begin{gathered}
S=\int d^{4} x \sqrt{-g}\left[-R+2(\nabla \phi)^{2}+e^{-2 \phi} F^{2}\right. \\
\left.+L\left(R_{i j k l}, F_{i j}, \phi\right)\right],
\end{gathered}
$$

where the Maxwell field $F_{i j}$ is associated with a $\mathrm{U}(1)$ subgroup of $E_{8} \times E_{8}$ or $\operatorname{Spin}(32) / Z_{2}$ and we set the remaining gauge fields to zero. $\phi$ is the dilaton field. The contribution of string theory to the classical gravitational action is through the function $L$. It is a perturbation expansion in inverse powers of the string tension parameter. The terms in this expansion may depend on all possible invariants constructed out of the curvature tensor $R_{i j k l}$, Maxwell field $F_{i j}$, dilaton field $\phi$, and their covariant derivatives. Under these assumptions, extremizing this action with respect to the $\mathrm{U}(1)$ potential $A_{i}$, dilaton field $\phi$, and the metric, we obtain the four-dimensional lowenergy limit of the string theory (extended gravitational field equations):

$$
\begin{aligned}
& G_{i j}-2 T_{i j}=E_{i j}, \\
& \nabla_{i}\left(e^{-2 \phi} F^{i j}\right)=E^{j}, \\
& \nabla^{2} \phi+\frac{1}{2} e^{-2 \phi} F^{2}=E .
\end{aligned}
$$

The energy-momentum tensor $T^{i j}$ corresponds to a Maxwell field coupled to the dilaton $\phi[9,10]$. The second-rank symmetric tensor $E_{i j}$, the vector $E_{i}$, and scalar $E$ coming from the variation of $L$ are the string correction terms to the classical gravitational field equations. They are believed to be composed of the curvature tensor $R_{i j k l}$, the Maxwell field $F_{i j}$, the dilaton field $\phi$, and of their covariant derivatives at all orders.

Although we consider the low-energy limit of string theory, our discussion in this paper applies to any theory derivable from a variational principle where the Lagrangian is an arbitrary smooth function of the Riemann tensor, Maxwell field tensor, dilaton field, and of their covariant derivatives.

The metric of a static and spherically symmetric space- 
time is given by

$$
d s^{2}=-A^{2} d t^{2}+B^{2} d r^{2}+C^{2}\left(d \theta^{2}+\sin ^{2} \theta d \phi^{2}\right),
$$

where $A, B$, and $C$ depend only on $r$. Our convention is as follows:

$$
R_{j k l}^{i}=\Gamma_{j l, k}^{i}+\Gamma_{m k}^{i} \Gamma_{j l}^{m}-(k \leftrightarrow l),
$$

$R_{i j}=R_{i k j}^{k}, R=R_{k}^{k}$. The Riemann tensor corresponding to this metric in a compact form is given by

$$
R_{i j k l}=g_{j l} S_{i k}-g_{j k} S_{i l}+g_{i k} S_{j l}-g_{j l} S_{k j}+\eta_{2} H_{i j} H_{k l},
$$

where

$$
S_{i j}=\eta_{0} M_{i j}+\eta_{1} k_{i} k_{j}+\frac{1}{2} \eta_{3} g_{i j}
$$

Here the scalars are given by

$$
\begin{aligned}
& \eta_{0}=\frac{C^{3}}{A B}\left[\frac{A_{, r} C-A C_{, r}}{B}\right]_{, r}, \\
& \eta_{1}=\frac{A B}{C}\left[\frac{C_{, r}}{A B}\right]_{, r}, \\
& \eta_{2}=C^{2}-\frac{C^{4}}{A B}\left[\frac{A_{, r} C-A C_{, r}}{B C}\right]_{, r}, \\
& \eta_{3}=-\frac{A, r}{A B_{, r}^{2} C} .
\end{aligned}
$$

The tensor $H_{i j}$ is antisymmetric and derivable from a vector potential $A_{i}$ :

$$
\begin{aligned}
A_{i} & =\cos (\theta) \delta_{i}^{\phi}, \\
H_{i j} & =\nabla_{i} A_{j}-\nabla_{j} A_{i} .
\end{aligned}
$$

The symmetric tensor $M_{i j}$ is defined as

$$
M_{i j}=H_{i}^{k} H_{k j}-\frac{1}{4} H^{2} g_{i j}
$$

where

$$
H^{2}=H^{i j} H_{i j} .
$$

The spacelike vector $k_{i}$ is given by

$$
k_{i}=\nabla_{i} r .
$$

The covariant derivatives of $H_{i j}$ and $k_{i}$ are given as

$$
\begin{aligned}
& \nabla_{l} H_{i j}=\rho\left(-2 k_{l} H_{i j}+k_{i} H_{j l}-k_{j} H_{i l}\right), \\
& \nabla_{i} k_{j}=\rho_{1} g_{i j}+\rho_{2} M_{i j}+\rho_{3} k_{i} k_{j},
\end{aligned}
$$

where

$$
\begin{aligned}
& \rho=\frac{C_{, r}}{C}, \\
& \rho_{1}=-\frac{A_{, r} C+A C_{, r}}{2 A B^{2} C}, \\
& \rho_{2}=C^{2} \frac{A, r}{A-A C_{, r}}
\end{aligned}
$$

$$
\rho_{3}=-\frac{(A B)_{, r}}{A B} \text {. }
$$

The covariant derivatives of $H_{i j}$ and $k_{i}$, as seen in Eqs. (17) and (18), are expressed only by themselves and the metric tensor. Hence, any higher-order covariant derivatives of these tensors must obey this rule. Since in Eq. (6) the Riemann tensor is given in terms of $H_{i j}, g_{i j}$, and $k_{i}$, and the scalars depending on $r$, its covariant derivatives at any order obey the same rule. Hence, we have the following theorem.

Theorem 1. Covariant derivatives of the Riemann tensor $R_{i j k l}$, the tensor $H_{i j}$, and the vector $k_{i}$ at any order are expressible only in terms of $H_{i j}, g_{i j}$, and $k_{i}$.

Since the contraction of $k^{i}$ with $H_{i j}$ vanishes, the only symmetric tensors constructable out of $H_{i j}, g_{i j}$, and $k_{i}$ are $M_{i j}$, the metric tensor $g_{i j}$, and $k_{i} k_{j}$. Then the following theorems hold.

Theorem 2. Any second-rank symmetric tensor constructed out of the Riemann tensor, the antisymmetric tensor $H_{i j}$, the dilaton field $\phi=\phi(r)$, and their covariant derivatives is a linear combination of $M_{i j}, g_{i j}$, and $k_{i} k_{j}$. Let this symmetric tensor be $E_{i j}^{\prime}$. Then we have

$$
E_{i j}^{\prime}=\sigma_{1} M_{i j}+\sigma_{2} g_{i j}+\sigma_{3} k_{i} k_{j},
$$

where $\sigma_{1}, \sigma_{2}$, and $\sigma_{3}$ are scalars which are functions of the metric functions, invariants constructed out of the curvature tensor $R_{i j k l}, H_{i j}$, and on the dilaton field.

Theorem 3. Any vector constructed out of the Riemann tensor $R_{i j k l}, H_{i j}$, the dilaton field $\phi=\phi(r)$, and their covariant derivatives is proportional to $k_{i}$. Let this vector be $E_{i}^{\prime}$. Hence, it reads

$$
E_{i}^{\prime}=\sigma k_{i}
$$

where $\sigma$ is a scalar as $\sigma_{1}, \sigma_{2}$, and $\sigma_{3}$.

We first discuss the solutions of the Einstein field equations. The Einstein tensor is found as

$$
\begin{aligned}
G_{i j}= & \left(2 \eta_{0}+\eta_{2}\right) M_{i j} \\
& -\frac{1}{2 B^{2} C^{4}}\left(6 B^{2} C^{4} \eta_{3}+B^{2} \eta_{2}-4 C^{4} \eta_{1}\right) g_{i j}+2 \eta_{1} k_{i} k_{j} .
\end{aligned}
$$

In the Einstein theory, the form of (25) gives us an idea about the form of the energy-momentum tensor as the source for the field equations. The source may include the electromagnetic field $F_{i j}$, a dilaton field $\phi$, and possibly a cosmological constant. In this case, the electromagnetic field may have an electric part in addition to the magnetic part, that is, $F_{i j}$ may have the form

$$
F_{i j}=e(r) \widetilde{H}_{i j}+q_{0} H_{i j},
$$

where $\widetilde{H}_{i j}$ is the dual of the tensor $H_{i j}$. In the general case, we have five equations for five functions $A, B, C$, $\phi(r)$, and $e(r)$. In the spherically symmetric case, the function $e(r)$ is not independent. Equation (3) forces us to choose it as

$$
e(r)=e_{0} \frac{A B}{C^{2}} e^{2 \phi},
$$


where $e_{0}$ is a constant. The remaining four functions $A$, $B, C$, and $\phi$ can be solved consistently. In fact, there are various types of solutions reported so far (see, for instance, [9]).

In the presence of string correction terms in Eqs. (2)-(4), the inclusion of the electric part is problematic. The dual $H_{i j}$ introduces a timelike vector $u_{i}=\delta_{i}^{t}$ into the tensor algebra discussed in theorem 1. This increases the number of symmetric tensors to five and number of vectors to two. Comparing the coefficients of these tensors and vectors in Eqs. (2) and (3), respectively, we obtain the equations for the metric functions $A, B, C, e(r)$, and the dilaton field $\phi=\phi(r)$. The total number of equations is raised to eight for these functions. In the Einstein case, since the right-hand sides of Eqs. (2)-(4) are absent, one gets a correct number of equations for these functions. In the case of the extended field equations it seems unlikely that this overdetermined system of ordinary differential equations has a solution.

In order to overcome this difficulty, we first let $e(r)=0$ and $F_{i j}=q_{0} H_{i j}$, where $q_{0}$ denotes the magnetic charge. In this case, the tensor $E_{i j}$ and the vector $E_{i}$ have exactly the same forms as $E_{i j}^{\prime}$ and $E_{i}^{\prime}$, respectively. Therefore, in the sequel we shall drop the primes over these tensors. By the theorems given above, we have now five equations for four functions. Again, the existence of a solution of this system of differential equations is not guaranteed. Hence, we further choose the electromagnetic field in such a way that the right-hand side of Eq. (3) automatically vanishes. This can be achieved in two ways: either $F_{i j}$ can be set equal to zero or $F_{i j}$ and $\phi$ can be taken covariantly constants. In the first case the Einstein field equations couple to a scalar field [1]. We have four equations and four unknown functions. Hence, we have a well-defined set of ordinary differential equations which may have exact solutions.

When the electromagnetic field is covariantly constant and $\phi=0$, Eq. (19) leads to $C=c_{0}=$ const. This simplifies the curvature functions $\eta_{0}, \eta_{1}, \eta_{2}$, and $\eta_{3}$. They read

$$
\begin{aligned}
& \eta_{1}=\eta_{3}=0, \\
& \eta_{2}=-\eta_{0}+c_{0}^{2}, \\
& \eta_{0}=\frac{c_{0}^{4}}{A B}\left[\frac{A, r}{B}\right)_{, r} .
\end{aligned}
$$

Since the dilaton field $\phi$ is set to zero, the vector $k_{i}$ does not show up in the set of tensors discussed in the above theorems. Hence, the tensor $E_{i j}$ is a linear combination of $M_{i j}$ and $g_{i j}$. This means that $\sigma_{3}=0$ in (2). The vector $E_{i}$ or $\sigma$ in Eq. (24) vanishes identically. Therefore, we have three equations for two functions $A, B$. This is again an overdetermined system. We shall further set $\eta_{0}=$ const. Under these assumptions, the extended field equations reduce to

$$
\begin{aligned}
\eta_{0}+c_{0}^{2}-2 q_{0}^{2} & =\sigma_{1}\left(\eta_{0}, c_{0}, q_{0} ; \alpha^{\prime}\right), \\
\frac{1}{2 c_{0}^{4}}\left(\eta_{0}-c_{0}^{2}\right) & =\sigma_{2}\left(\eta_{0}, c_{0}, q_{0} ; \alpha^{\prime}\right),
\end{aligned}
$$

$$
2 \frac{q_{0}^{2}}{c_{0}^{4}}=E\left(\eta_{0}, c_{0}, q_{0} ; \alpha^{\prime}\right),
$$

where $\alpha^{\prime}$ is the inverse string parameter. Here we have three algebraic equations for three constants $c_{0}, \eta_{0}$, and the constant $q_{0}$. The scalars $\sigma_{1}, \sigma_{2}$, and $E$ are now also constants depending upon $c_{0}, \eta_{0}$, and $q_{0}$, and on the parameters of the theory under consideration, such as the string tension. Since $\eta_{0}$ is a constant, Eq. (30) is exactly soluble. It reads

$$
B=\frac{A_{, r}}{\sqrt{\left(\eta_{0} / c_{0}^{4}\right) A^{2}+a_{1}}},
$$

where $a_{1}$ is a constant. This is the only equation which determines the metric functions $A$ and $B$. Although the function $A$ appears to be arbitrary, by changing $r, B$ can be set to any function of $r$. We choose it as $1 / A$. With this choice of $B$, the above differential equation can be integrated easily and we determine the spacetime metric as

$d s^{2}=-A^{2} d t^{2}+\frac{1}{A^{2}} d r^{2}+c_{0}^{2}\left(d \theta^{2}+\sin ^{2} \theta d \phi^{2}\right)$,

where

$$
A^{2}=r\left(\frac{\eta_{0}}{c_{0}^{4}} r+a_{1}\right)+a_{2}^{2} .
$$

Here $a_{1}$ and $a_{2}$ are constants. According to whether $\eta_{0}$ is zero or not there are two distinct solutions.

Type (a):

$$
\eta_{0}=a_{1}=0, \quad A^{2}=1 \text {. }
$$

It is a direct product of $S^{2}$ and a two-dimensional flat Minkowski space. The vector $k_{i}$ is covariantly constant; hence, it is also a spacelike Killing vector.

Type (b):

$$
a_{2}=0, \quad A^{2}=r\left(\frac{\eta_{0}}{c_{0}^{4}} r+a_{1}\right) .
$$

This metric describes a spacetime which is a direct product of a two-dimensional pseudosphere and $S^{2}$.

We now state that the metrics given above are exact solutions of the gravitational field equations with string corrections. They are all nonsingular and homogeneous spacetimes. These metrics are also the solutions of the Einstein-Maxwell equations. For instance, the second solution [type (b)] with

$$
\eta_{0}=c_{0}^{2}=q_{0}^{2}
$$

is the Levi-Civita Bertotti-Robinson metric. Under the string corrections the form of these metrics is preserved but the parameters appearing in the metrics no longer satisfy the above equations (39). In this case the relations among these constants are given by Eqs. (31)-(33).

The above metrics, in particular type (b), have interesting features. They describe the geometry of black-hole solutions in the neighborhood of their outer horizons. These regions are the throats of the Einstein-Rosen bridges of the corresponding hole solutions. For exam- 
ple, the Levi-Civita Bertotti-Robinson universe is known to be the throat of the extreme charged black hole [14-16]. Here, extending the same idea let us find the metric describing the region near the outer horizons of the charged black holes in de Sitter space [17]. In the spherically symmetric case such a black-hole solution is given by

$d s^{2}=-W^{2} d t^{2}+\frac{1}{W^{2}} d r^{2}+r^{2}\left(d \theta^{2}+\sin ^{2} \theta d \phi^{2}\right)$,

where

$$
W^{2}=1-\frac{2 m}{r}+\frac{Q^{2}}{r^{2}}-\lambda_{0} r^{2} .
$$

Here $m, Q$, and $\lambda_{0}$ are, respectively, the mass, charge, and the cosmological constant. In the neighborhood of the outer horizon $r=r_{h}+\epsilon$, where $W\left(r=r_{h}\right)=0$, the metric and the function $W$ take the form

$$
\begin{aligned}
& d s^{2}=-W^{2} d t^{2}+\frac{1}{W^{2}} d \epsilon^{2}+r_{h}^{2}\left(d \theta^{2}+\sin ^{2} \theta d \phi^{2}\right), \\
& W^{2}=\epsilon(\alpha \epsilon+\beta) .
\end{aligned}
$$

This metric remains to be the solution of the EinsteinMaxwell field equations with a cosmological constant provided

$$
m=\frac{Q^{2}}{r_{h}},
$$

and $\alpha, \beta$, and $\lambda_{0}$ are given by

$$
\begin{aligned}
& \alpha=\frac{1}{r_{h}^{4}}\left(2 Q^{2}-r_{h}^{2}\right), \\
& \beta=\frac{2}{r_{h}^{3}}\left(-r_{h}^{2}+Q^{2}\right), \\
& \lambda_{0}=\frac{2}{r_{h}^{4}}\left(r_{h}^{2}-Q^{2}\right) .
\end{aligned}
$$

Notice that the cosmological constant is no longer an independent parameter. This limiting metric (42) is exactly of type (b) given above with the identifications

$$
\begin{aligned}
& \frac{\eta_{0}}{c_{0}^{4}}=\alpha, \quad a_{1}=\beta, \\
& c_{0}=r_{h}, \quad q_{0}=Q .
\end{aligned}
$$

The extended field equations (31)-(33) alter the definitions given in Eqs. (44)-(47) of $\alpha, Q, r_{h}$, and $\lambda_{0}$. By the utility of this identification they now satisfy

$$
\begin{aligned}
& r_{h}^{4} \alpha+r_{h}^{2}-2 Q^{2}=\sigma_{1}\left(\alpha, r_{h}, Q ; \alpha^{\prime}\right), \\
& \frac{1}{2 r_{h}^{2}}\left(r_{h}^{2} \alpha-1\right)=\sigma_{2}\left(\alpha, r_{h}, Q ; \alpha^{\prime}\right),
\end{aligned}
$$

$$
2 \frac{Q^{2}}{r_{h}^{4}}=E\left(\alpha, r_{h}, Q ; \alpha^{\prime}\right)
$$

Hence, the above equations relate the mass and the charge to the string tension. The type (a) solution corresponds to the throat of the extreme charged hole with dilaton discussed recently in [9].

We conclude that the regions in the neighborhood of the outer horizons of the charged black holes are preserved under string corrections. String theory only changes the relations among the parameters $\alpha, Q, r_{h}$, and the cosmological constant $\lambda_{0}$. They are related to the inverse string tension parameter $\alpha^{\prime}$ through Eqs. (50)-(52).

We have found two distinct metrics which may constitute exact solutions of the gravitational field equations with string correction terms at all orders. The existence of solutions, of course, depends on the functional dependencies of $\sigma_{1}, \sigma_{2}$, and $E$ on the parameters $c_{0}, \eta_{0}, q_{0}$ or on $r_{h}, \alpha$, and $Q$. Since the extended field equations or the explicit forms of $E_{i j}, E_{i}$, and $E$ are not known yet, it is not possible to give an answer to this existence problem. On the other hand, the gravitational field equations with the quadratic curvature terms, such as the Gauss-Bonnet term, are commonly known to be the one-loop corrections. The tensors $E_{i j}, E_{i}$, and $E$ in this case are simpler and Eqs. (31) - (33) have consistent solutions.

We have shown that the metrics that solve the extended field equations are asymptotic forms of the charged nonrotating black holes of the Einstein theory. The mass and the charge are related to string tension. When we consider only the first-order corrections, the tensor $E_{i j}$ is the Gauss-Bonnet term which vanishes identically for both types of metrics we found in this paper. The effect of string corrections at this order comes from Eq. (33). When the cosmological constant is set to zero, the mass, charge, and radius of the horizon turn out to be equal and they are proportional to $\sqrt{\alpha^{\prime}}$. Hence, at the Planck scale we have an exact solution of the extended field equations which is the signature of a classical black hole. It is exactly the throat of the extreme charged black hole.

Inclusion of the three-form field into the field equations (2) $-(4)$ is also possible. This will not alter our conclusion. Let $H_{i j k}=\mu k_{[i} H_{j k]}$, where $\mu$ is a function of $r$. One can prove the following theorem: The only antisymmetric second-rank tensor obtainable out of $R_{i j k l}, H_{i j}, k_{i}, H_{i j k}$, and their covariant derivatives is proportional to $H_{i j}$. Hence, in addition to the field equations (3) and (4), we have $\nabla_{i}\left(e^{-2 \phi} H^{i j k}\right)=\sigma_{4} H^{j k}$, where $\sigma_{4}$ is a scalar as $\sigma_{1}, \sigma_{2}$, $\sigma_{3}$. Letting $H_{i j}$ be covariantly constant, $\phi=\phi_{0}=$ const and $\mu=0$, we have (31)-(33) with $\sigma$ 's also depending on $\phi_{0}$. In addition to these equations we also have $\sigma_{4}\left(\eta_{0}, c_{0}, q_{0}, \phi_{0} ; \alpha^{\prime}\right)=0$. This leads to four algebraic equations for four constants $\eta_{0}, c_{0}, q_{0}$, and $\phi_{0}$.

Extension of the results reported in this paper to higher dimensions is also possible. For instance, a $D$ dimensional spacetime, which is a direct product of a two-dimensional pseudosphere and $S^{D-2}$, is a solution of Einstein-Maxwell field equations in $D$ dimensions. The Maxwell field is covariantly constant. It is possible to show that the Riemann tensor has exactly the same form 
as given in Eq. (6) with slight changes in the definitions of $\eta_{0}, \eta_{1}, \eta_{2}$, and $\eta_{3}$. The theorems given in this work remain valid. The form of the metric is preserved under string corrections. The extended field equations are again redefinitions of the parameters appearing in the theory.
I would like to thank Rahmi Güven for a critical reading of the manuscript and for several suggestions and Yavuz Nutku for discussions. This work was partially supported by the Scientific and Technical Research Council of Turkey (TUBITAK).
[1] C. G. Callan, R. C. Myers, and M. J. Perry, Nucl. Phys. B311, 673 (1988).

[2] D. G. Boulware and S. Deser, Phys. Rev. Lett. 55, 2656 (1985).

[3] J. T. Wheeler, Nucl. Phys. B268, 737 (1986).

[4] J. T. Wheeler, Nucl. Phys. B273, 732 (1986).

[5] D. L. Wiltshire, Phys. Lett. 169B, 36 (1986).

[6] R. C. Myers, Nucl. Phys. B289, 701 (1987).

[7] R. C. Myers, Phys. Rev. D 36, 392 (1987).

[8] G. W. Gibbons and K. Maeda, Nucl. Phys. B298, 741 (1988).

[9] D. Garfinkle, G. T. Horowitz, and A. Strominger, Phys.
Rev. D 43, 3140 (1991).

[10] N. R. Stewart, Class. Quantum Grav. 8, 1701 (1991).

[11] R. Güven, Phys. Lett. B 191, 275 (1987).

[12] D. Amati and C. Klimčik, Phys. Lett. B 219, 443 (1989).

[13] G. Horowitz and A. R. Steif, Phys. Rev. Lett. 64, 260 (1990).

[14] B. Carter, in Black Holes, edited by C. DeWitt and B. DeWitt (Gordon and Breach Science, New York, 1973).

[15] C. Misner, K. S. Thorne, and J. A. Wheeler, Gravitation (Freeman, San Francisco, 1973).

[16] D. L. Wiltshire, Phys. Rev. D 31, 2445 (1988).

[17] P. C. Davies, Report No. NCL-89 TP9 (unpublished). 